\title{
- Teledentistry: An Update
}

\section{Dr. Little Mahendra}

IJCRR

Section: Healthcare

ISI Impact Factor

(2019-20): 1.628

IC Value (2019): 90.81

SJIF (2020) $=7.893$

(c) (7) (3)

Copyright@IJCRR
MDS, PhD, Postdoc (USA), MFDS (Glasgow), FIMSA, PGDHM, Dean, Maktoum Bin Hamdan Dental University College, Dubai.

Email:dean@mbhduc.org
The current scenario has had a major impact on the dental profession since the initial lockdown till date. According to many surveys conducted around the globe, the largest impact of the pandemic on dental practices has been the drop in revenue. The dental practice was unable to sustain during the initial lockdown, that is when revenue was hit the most. However, as we approach nearly a full year of living with the pandemic, it has been proven that dentistry is an essential service and can be provided up to some extend by teledentistry as well.

During the lockdown, many of the dentists have setup telehealth services to provide virtual care for their patients. This platform is able to collect and analyze the data allowing the dentist to remotely evaluate the patient's issues and provide them with essential advice and treatment options. During the teledentistry appointments, the patient's problem can be analyzed with the help of videos and pictures, and urgent care evaluations can be provided with pharmacologic management or follow-up care after emergency visits.
Tele-dentistry was not a platform which was used frequently by the dentists before, thinking that dental care always requires in-person visits. But, in the present times, the teledentistry mainly focuses on how this platform can be used in public health and during the time of lockdowns when it becomes difficult for the dentists and patients to be present in the dental office.

While many dentists believe revenue decreases will continue to be a great challenge in the coming year, we can at least feel safe that dental offices can sustain the pressure of lockdowns with the help of teledentistry and can remain open to continue treating patients in emergency.

While the pandemic has necessitated the use of this platform, more research is needed to help determine the technology needs, criteria for reimbursement, and types of problems that can be addressed during teledentistry. 